\section{Factors influencing quality of life and predictors of low quality of life scores in patients on treatment for pulmonary tuberculosis: a cross sectional study}

\author{
Olufunke O. Adeyeye, ${ }^{1}$ \\ Olayinka O. Ogunleye, ${ }^{1,2}$ Ayodele Coker, ${ }^{3}$ \\ Yetunde Kuyinu, ${ }^{4}$ Raymond T. Bamisile, ${ }^{1}$ \\ Udeme Ekrikpo, ${ }^{5}$ Babatunde Onadeko' \\ 'Department of Medicine, Lagos State \\ University College of Medicine and \\ Teaching Hospital, Ikeja, Lagos; \\ 2Department of Pharmacology, Lagos \\ State University College of Medicine, \\ Ikeja, Lagos; ${ }^{3}$ Department of Behavioural \\ Medicine, Lagos State University College \\ of Medicine, Ikeja, Lagos; ${ }^{4}$ Department of \\ Community Medicine \& Primary Health \\ Care, Lagos State University, College of \\ Medicine, Ikeja, Lagos; ${ }^{5}$ Department of \\ Medicine, University of Uyo Teaching \\ Hospital, Uyo, Nigeria
}

\section{Abstract}

There is paucity of information on the quality of life of patients with pulmonary tuberculosis in Nigeria. This study assessed the factors influencing their quality of life and the independent predictors of low quality of life scores.

Two hundred and sixty consecutive patients with pulmonary tuberculosis seen at the Lagos University Teaching Hospital were evaluated for health related quality of life using the World Health Organization Quality of life instrument (WHOQoL-BREF). Sociodemographic characteristics of the patients were related to the various domains of quality of life and a multivariate logistic regression analysis was performed to identify the independent predictors of low quality of life scores in the patients.

The mean age of the patients was $36.7 \pm 12$ years. Sex, age and marital status of patients were found to influence quality of life scores. The independent predictors of low quality of life scores were low monthly income, duration of the illness, concomitant illnesses, unemployment, advancing age and male gender. Several socio demographic and economic factors influenced the quality of life of patients with tuberculosis and are predictive of poor scores. It is important to consider these factors when treating patients with tuberculosis to optimise outcome of care.

\section{Introduction}

Quality of life (QoL) is a broad, complex, multidimensional concept that incorporates psychological, economic, philosophical, socio-cultural and spiritual dimensions. Its definition and measurements are surrounded by controversies. ${ }^{1}$

Studies done in patients with different chronic diseases like chronic renal failure, acquired immunodeficiency syndrome and psychiatry disorders had shown that different social and demographic factors affects quality of life of these patients. ${ }^{2-5}$ Likewise, there are available literature showing significant adverse effects of tuberculosis on quality of life of patients. ${ }^{6-8}$ These studies were however carried out largely outside black Africa. There is currently no study in the public domain on the quality of life of Nigerian patients with tuberculosis. Only few of the available literature on this subject have identified the predictors of the well-established poor quality of life outcomes among the patients with tuberculosis. This report is part of a self-sponsored, researchers initiated study that examined the quality of life of patients on treatment for pulmonary tuberculosis alongside adherence to therapy and adverse drug effects. It is aimed at identifying the factors influencing the quality of life of patients on treatment for pulmonary tuberculosis and the predictors of low quality of life scores in these patients. This is considered a needful step towards instituting a more wholistic care of pulmonary tuberculosis patients for better outcome in a population with high burden of the disease.

\section{Materials and Methods}

The study was conducted at the chest unit of Lagos State University Teaching Hospital, Ikeja, Lagos (LASUTH) between May 2009 and September 2009. The hospital chest clinic receives an average of twelve new cases of tuberculosis per week and about fifty newly diagnosed with tuberculosis monthly. At the commencement of the study there were about two hundred and fifty-five patients receiving treatment out of which about one hundred and sixty nine were on directly observed therapy.

Ethical approval for the study was obtained from the Ethics and Research Committee of the hospital and all study participants gave written informed consent.

Inclusion criteria were any patient with diagnosis of pulmonary tuberculosis either by sputum smear or culture or radiological features and registered for anti-tuberculosis treatment at the chest clinic of LASUTH. The protocol and criteria for diagnosing and treating tuberculosis
Correspondence: Olayinka Olabode Ogunleye, Department of Pharmacology, Lagos State University College of Medicine, 1-5, Oba Akinjobi Street, Ikeja GRA, Lagos, Nigeria.

Tel.: +234.805.335.7057

E-mail: yinkabode@yahoo.com

Key words: quality of life, tuberculosis, Nigeria.

Contributions: 00A, conceived the study, designed the study, carried out data acquisition, involved in data analysis and interpretation and revised the manuscript; 000, conceived the study, designed the study, carried out data analysis and interpretation, drafted and revised the manuscript; UE, involved in data analysis, interpretation of results and revision of the manuscript; AC, YA, RTB and B0, participated in data collection and revision of the manuscript. All authors read and approved the final manuscript.

Conflict of interests: the authors declare no potential conflict of interests.

Received for publication: 26 August 2013.

Revision received: 5 January 2014.

Accepted for publication: 16 July 2014.

This work is licensed under a Creative Commons Attribution NonCommercial 3.0 License (CC BYNC 3.0).

(C) Copyright O.O. Adeyeye et al., 2014

Licensee PAGEPress, Italy

Journal of Public Health in Africa 2014; 5:366

doi:10.4081/jphia.2014.366

were based on the National Tuberculosis Control Policy of the Federal Republic of Nigeria, which was adapted from the World Health Organization (WHO) documents. ${ }^{9}$

The data collection tool was a structured questionnaire which had four sections; A, B, C and $\mathrm{D}$ respectively. Section A sought information on the socio-demographic characteristics of participants including age, sex, educational and employment status, religion and estimates of income and expenditure. Section B obtained information on the clinical history, which included symptoms at presentation, alcohol use, smoking, co-morbidities, concomitant medications and adverse effects to drugs. Section C obtained information on adherence to therapy while Section D incorporated the assessment of the quality of life of participants using the World Health Organization (WHO) QoL BREF parameters. ${ }^{10}$

The WHOQoL-BREF is an abbreviated version of the WHOQoL-100 instrument consisting of 26 questions, which assesses four specific domains of well-being namely physical health (domain 1), psychological health (domain 2), social relationship (domain 3 ) and the environment (domain 4). Domain 1 incorporates the following facets: activities of daily living, 
dependence on medicinal substances and medical aid, energy and fatigue, mobility, pain and discomfort, sleep and rest, work capacity. Domain 2 assesses bodily image and appearance, negative feelings, positive feelings, selfesteem, spirituality/religious/personal beliefs, thinking, learning, memory and concentration. Domain 3 assesses personal relationships, social supports and sexual activities while domain 4 incorporates the following facets as well: financial resources, freedom/physical safety/security, accessibility and quality of health and social care, home environment, opportunity for acquiring new information and skills, participation in and opportunities for recreation and leisure activities, physical environment (pollution/noise/traffic/climate) and transport. Domain scores were scaled in a positive direction of 1 to 5 with 5 being the most optimistic response. The four domain scores denote an individual's perception of quality of life in each particular domain. Higher scores of 4 and 5 indicated a higher quality of life while lower scores below 3 indicated a low quality of life.

The study tool was pre-tested on thirty patients with tuberculosis being managed in a separate public health facility and the necessary corrections based on the responses obtained were made. The corrections made included precoding the income and expenditure sections into various ranges of figures, which was the preferred mode of respondents in disclosing their financial status as against the initial open-ended questions. Also, provision was made in the lists of symptoms and potential adverse drug reactions for participants to state items that were applicable to them but not captured in the instrument. The instrument was thereafter administered at the study site to consecutive patients who meet the inclusion criteria and had given written informed consent by the investigators assisted by three other trained facilitators who were house officers rotating through the chest unit of LASUTH during the study period.

All information collected from each respondent was entered into an IBM compatible computer and analyzed using commercially available statistical package Stata version 10 (STATA Corp., College Station, TX, USA).

Continuous variables were expressed as means (standard deviation), categorical variables as proportions. The mean score of items in each domain of wellbeing described above was used to calculate the domain score. Mean scores were then multiplied by 4 in order to make the domain scores compatible with the scores used in WHOQoL-100. Following the transformation scales obtainable in the WHOBREF manual, the domain scores were transformed to a $0-100$ scale. $^{11}$

A multivariate logistic regression analysis was performed to identify the independent predictors of low quality of life scores in the patients among the socio-demographic and economic factors. A low quality of life score in accordance with WHO-BREF guideline was taken as a raw score of 21 or less in domain 1 , 18 or less in domain 2,9 or less in domain 3 and 24 or less in domain 4 . These cut offs scores translates to a transformed score of $\mathbf{5 0}$ or less in all the domains. All statistical tests were carried out at $5 \%$ level of significance.

\section{Results}

Two hundred and sixty patients receiving treatment for tuberculosis took part in the study out of a total of three hundred and twenty five patients identified as potential participants during the study period. The mean age of respondents was $36.7 \pm 12.6$ years. There were 190 males (73.1\%) and 70 females (26.9\%). Figures 1, 2 and 3 show the marital status by percentages, the educational levels attained and the employment status of the studied population respectively.

The most prevalent co-morbidity was HIV/AIDS; 40 (14.5\%), followed by hypertension; 15 (5.8\%). Others were diabetes mellitus; $10(3.8 \%)$, both asthma and seizure disorder with prevalence of $5(1.9 \%)$ respectively.

Majority of the patients, $229(88.1 \%)$ had never smoked cigarette. Only 26 (10\%) of the patients studied were current smokers while 5

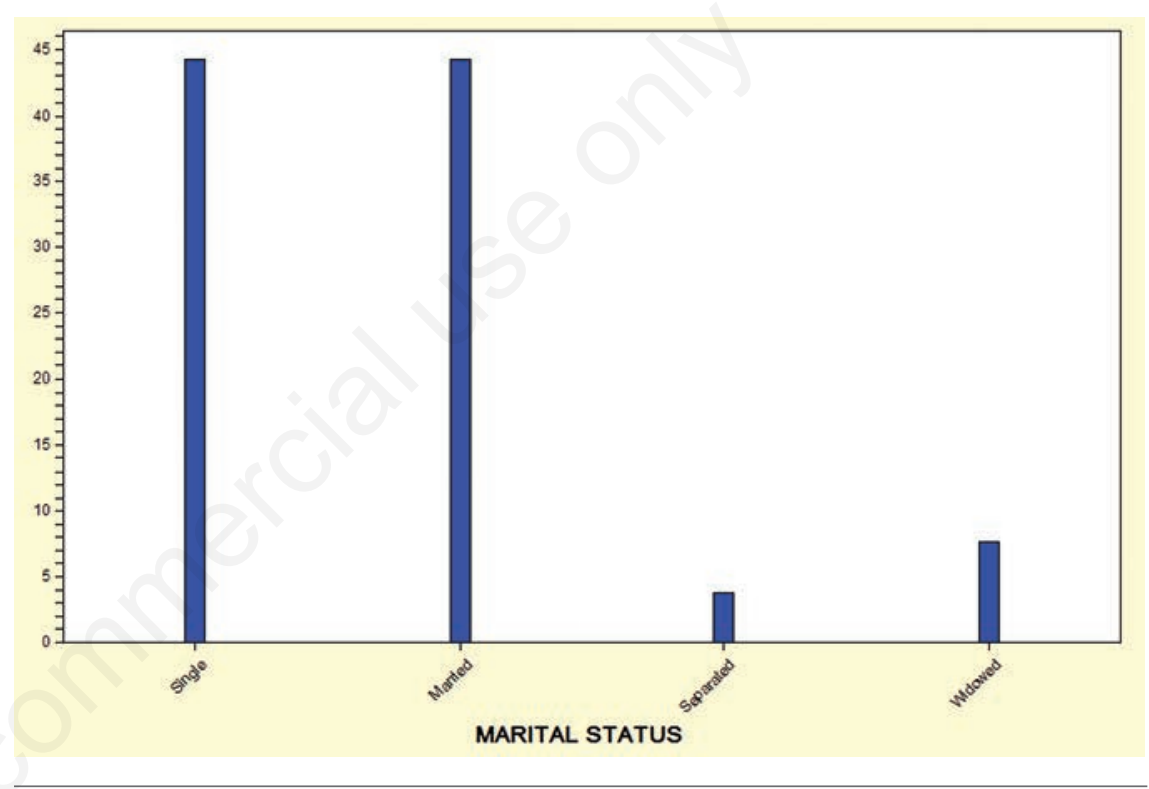

Figure 1. Marital status by percentages of studied population.

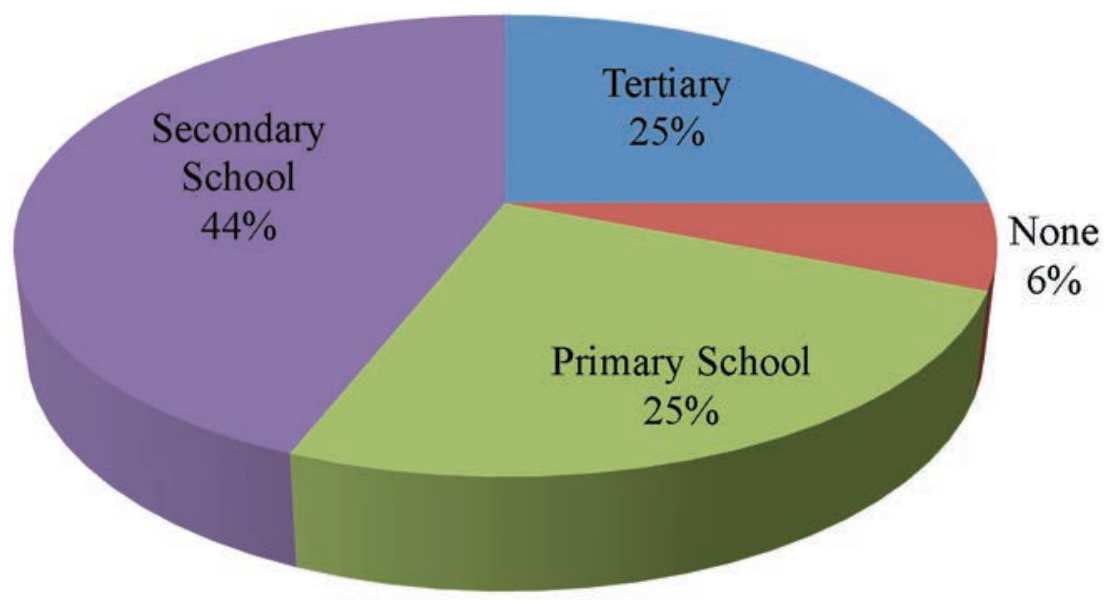

Figure 2. Highest educational levels attained by percentages of the population studied. 
(1.9\%) of the patients were ex-smokers. Only $50(19.2 \%)$ of the participants were current alcohol users, 210 (80.8\%) did not use alcohol.

The highest mean transformed quality of life score was recorded in social relationship domain of health $(70.80 \pm 18.12)$ while the lowest was in the environmental domain (66.33 \pm 15.20). The mean transformed quality of life scores in the other domains of health included $67.40 \pm 22.20$ in the physical and $66.75 \pm 16.60$ in the psychological domains respectively.

The mean quality of life scores in the various domains of health were compared by sexes, age ranges ( $<50$ years and $\geq 50$ years) and the marital status as shown in Table 1 . Both males and females did not differ with respect to the physical and environmental domains but the females had better quality of life in the psychological and social relationship aspects of quality of life. With the exemption of the environmental domain of health, younger age group ( $<50$ years) had superior health related quality of life in all other domains of health. The quality of life score in the physical domain was better in individuals with spouses compared with those without. In contrast, those without spouses fared better in the psychological domain of health than those with spouses. There were no differences in the social and environmental domains of quality of health with respect to marital status.

Tables 2-5 summarize the final multivariate logistic models for the independent predictors of low quality of life scores in the various domains. The predictors of low scores in the physical domain of health were low level of monthly income less than 14,999 naira (about US\$ 93) relative to higher monthly incomes, being on treatment for other conditions, the duration of the illness and unemployment.

The predictors of low scores in the psychological domain of health were age, male gender, low level of monthly income less than 14,999 naira (about US\$ 93) and the duration of the illness. The predictors for low scores in both the social relationship and environmental domains of quality of health were mainly low monthly incomes less than 14,999 naira (about US\$ 93) and duration of illness as shown in Tables 4 and 5 respectively.

\section{Discussion}

Tuberculosis studies have concentrated on clinical outcomes, only few studies have examined the impact of tuberculosis on patients' quality of life. In the past few decades there has been a reawakening of the principle that health is a fundamental human right and a worldwide social goal that is essential to the satisfaction of basic human need. It is therefore very important to improve quality of life even in diseased states.

In line with previous findings by several workers in some parts of Africa and Asia that there is a higher prevalence of tuberculosis among men than women, ${ }^{12-15}$ there were more males than females in the present population studied in a ratio of $2.7: 1$. While there were more males than females affected by this disease in this current study, both sexes fared comparably in the physical and environmental domains of health (Table 1) but the females had superior psychological and social relationship indices of quality of health. Muniyandi $e t$ al. using the SF 36 instrument of quality of life evaluation in a South Indian population had reported lower scores in females compared with males in the mental and social domains in contrast to our present findings. ${ }^{16}$ This perhaps might be outcomes occasioned by either socio-cultural differences between the popula- tions studied or the differences in the instruments used.

Within the Nigerian black population studied, the socio-cultural norm is a bit repressive regarding the expression of views and feelings by the female gender. The presumption is that women are to be seen more and heard less, thus potentially causing poor expressions of their views and feelings. Perhaps, this may have precluded the disclosure of their real states of health. On the positive note however, the observed superiority of the psychological and social health status in females in this study may be a reflection of a better coping skill by the females in dealing with the pressure and challenges of the disease. The female gender within the Nigerian society in the contemporary times appears to be much given to issues of spirituality, religiosity, social networking and interpersonal relationships. These factors are key determinants of social and psychological domains of quality of health. The greater disposition of women to spirituality as a means of obtaining respite in disease states and its positive influence on their health have been documented by other workers outside the Nigerian communities. ${ }^{17,18}$

In contrast, the lower scores in males in the social relationship domain may be reflective of the greater sensitivities of males towards matters affecting their personal rela-

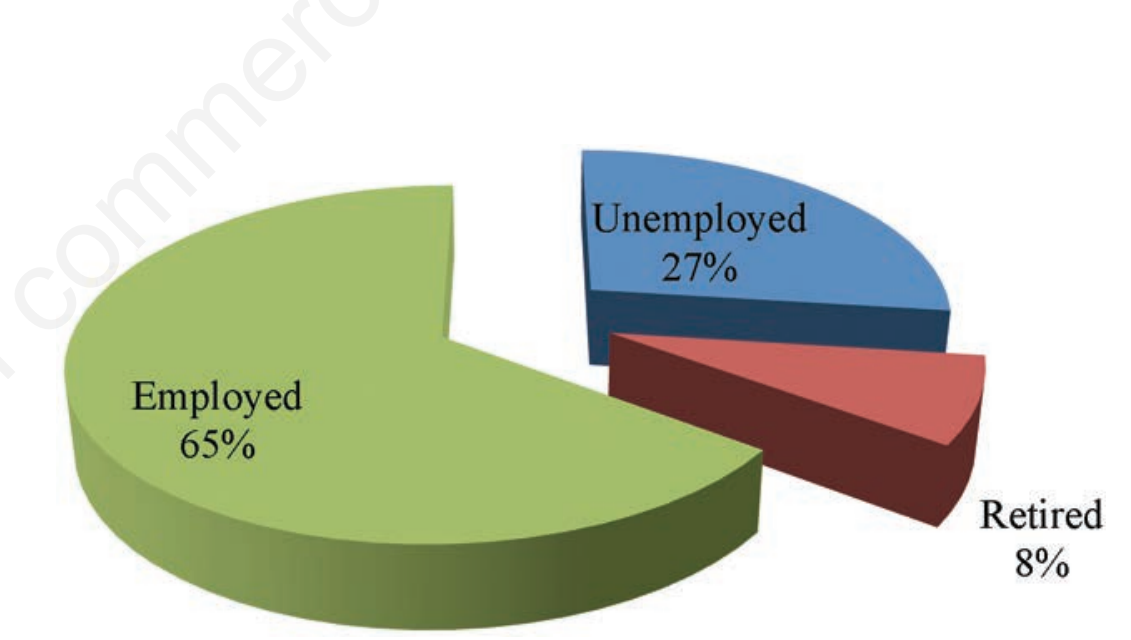

Figure 3. Employment status of studied population by percentages.

Table 1. Sex, age range and marital status based comparisons of mean quality of life scores in health domains.

\begin{tabular}{|c|c|c|c|c|c|c|}
\hline \multirow[t]{2}{*}{ Domain of gender } & \multicolumn{2}{|c|}{ Gender } & \multicolumn{2}{|c|}{ Age range } & \multicolumn{2}{|c|}{ Spouse } \\
\hline & Male & Female & $<50$ years & $>50$ years & With & Without \\
\hline Physical & $67.03 \pm 22.7$ & $68.46 \pm 20.8$ & $72.98 \pm 16.7$ & $50.14 \pm 21.3^{*}$ & $71.71 \pm 18.0$ & $63.90 \pm 24^{\circ}$ \\
\hline Psychol & $64.00 \pm 17.3$ & $73.58 \pm 12.2^{*}$ & $72.98 \pm 16.7$ & $59.86 \pm 7.9^{\#}$ & $64.59 \pm 18.1$ & $69.48 \pm 14.1$ \\
\hline Social & $67.53 \pm 18.2$ & $79.89 \pm 14.8^{*}$ & $72.98 \pm 16.7$ & $55.17 \pm 20.7^{*}$ & $69.27 \pm 19.8$ & $72.47 \pm 16.0$ \\
\hline Environ & $65.12 \pm 15.0$ & $69.38 \pm 16.4$ & $67.05 \pm 15.5$ & $61.50 \pm 12.4$ & $69.27 \pm 19.8$ & $67.67 \pm 12.5$ \\
\hline
\end{tabular}

Psychol, psychological domain; Environ, environmental domain. ${ }^{*} \mathrm{P}<0.0001 ;{ }^{\circ} \mathrm{P}=0.005 ;{ }^{*} \mathrm{P}<0.01$. 
tionships and sexual activities. Since men are generally perceived as strong while women are reckoned as the weaker sex within the population studied, men are less likely to receive social support in disease states than women. The poorly developed formal or organized social support service for the sick in Nigeria at present is also a possible contributory factor to these observations. Social supports for the sick presently are almost limited to those provided by members of the immediate family of the sick and some religious organizations with unpredictable availability.

In this study, the lower age group $(<50$ years) had better quality of life in the physical, social relationship and psychological domains of health compared with those above the age of 50 years. These findings were in agreement with that of Muniyandi et al. ${ }^{16}$ with the exception of the social domain where no difference existed between the old and the young age groups. It is however worthy to note that the age cut-offs applied differ between these two studies and the instruments used also comprised of varied parameters. A possible explanation for these observations among our study population may be the fact that advancing age is often associated with degenerative changes and several other co-morbid conditions, which may significantly impair the quality of life of the patients. The younger patients being more economically active are likely to have better financial resources to access care, better quality of home environment, good social relationships and achievement of better self-esteem. These are likely to improve their long-term health outcomes when compared with the older patients. The younger age group is also more likely to have a better understanding of disease processes and better compliance with treatment modalities. These are additional factors that could potentially explain better quality of life in this age category.

In this report, almost $56 \%$ of the population studied was without spouses (single, separated or widowed). Those without spouses had a higher score in the psychological domain while those with spouses fared better in the physical domain of health related quality of life but both categories did not differ in the social and environmental domains respectively. The possible reasons for these observations cannot be speculated immediately. They may be as a result of multiplicity of factors requiring further evaluation.

There has been a consistent demonstration of poor quality of life associated with tuberculosis in practically all domains of health as well as improvements with treatment but only few studies have attempted to identify the factors predicting these established facts. $., 7,15,16,19,20$ This present study attempted filling this
Table 2. Predictors of low scores in the physical domain of health related quality of life.

\begin{tabular}{lcc} 
Factor & Odds ratio $(95 \% \mathrm{CI})$ & P value \\
Age & $0.97(0.91-1.02)$ & 0.267 \\
Gender & $0.56(0.19-1.59)$ & 0.280 \\
\hline Religion & $0.59(0.22-1.60)$ & 0.303 \\
Income & $0.16(0.06-0.42)$ & $<0.001^{*}$ \\
\hline Alcohol & $2-33(0.54-10.00)$ & 0.255 \\
Other treatment & $0.09(0.02-0.48)$ & $0.004^{*}$ \\
\hline Duration & $1.08(1.04-1.13)$ & $<0.001^{*}$ \\
Co_HIV & $1.73(0.54-5.51)$ & 0.352 \\
\hline Employment & $0.13(0.31-0.49)$ & $0.003^{*}$
\end{tabular}

$\mathrm{CI}$, confidence interval; Other treatment, on treatment for other condition; Co_HIV, HIV co-infection. ${ }^{*} \mathrm{P}<0.05$.

Table 3. Predictors of low scores in the psychological domain of health related quality of life.

\begin{tabular}{lcc} 
Factor & Odds ratio $(95 \% \mathrm{CI})$ & P value \\
Age & $0.28(0.11-0.76)$ & $0.012^{*}$ \\
Gender & $0.04(0.00-0.51)$ & $0.014^{*}$ \\
\hline Income & $0.11(0.01-0.89)$ & $0.039^{*}$ \\
Duration & $0.93(0.86-0.99)$ & $0.049^{*}$
\end{tabular}

$\mathrm{Cl}$, confidence interval. $* \mathrm{P}<0.05$.

Table 4. Predictors of low scores in the social relationship domain of health related quality of life.

\begin{tabular}{lcc}
\hline Factor & Odds ratio $(95 \%$ CI $)$ & P value \\
Age & $0.91(0.81-0.02)$ & 0.097 \\
Gender & $1.21(0.28-5.33)$ & 0.792 \\
\hline Marital status & $0.86(0.09-8.34)$ & 0.896 \\
Employment status & $0.33(0.07-1.41)$ & 0.133 \\
\hline Religion & $0.75(0.07-8.07)$ & 0.815 \\
Income & $0.09(0.02-0.32)$ & $<0.001^{*}$ \\
\hline Alcohol & $0.15(0.02-1.44)$ & 0.100 \\
Other treatment & $0.39(0.07-2.17)$ & 0.287 \\
\hline Duration & $1.14(1.06-1.23)$ & $<0.001^{*}$ \\
Co_HIV & $0.39(0.09-1.65)$ & 0.205
\end{tabular}

CI, confidence interval; Other treatment, on treatment for other condition; Co_HIV, HIV co-infection. *P $=<0.05$.

Table 5. Predictors of low scores in the environmental domain of health related quality of life.

\begin{tabular}{lcc} 
Factor & Odds ratio $(95 \% \mathrm{CI})$ & P value \\
Age & $0.91(0.81-1.02)$ & 0.097 \\
Gender & $1.22(0.28-5.33)$ & 0.792 \\
\hline Marital status & $0.86(0.09-8.34)$ & 0.896 \\
Employment status & $0.32(0.07-1.41)$ & 0.133 \\
\hline Religion & $0.75(0.07-8.07)$ & 0.815 \\
Income & $0.09(0.02-0.32)$ & $<0.001^{*}$ \\
\hline Alcohol & $0.15(0.02-1.44)$ & 0.100 \\
Other treatment & $0.39(0.07-2.17)$ & 0.287 \\
\hline Duration & $1.14(1.06-1.23)$ & $<0.001^{*}$ \\
Co_HIV & $0.39(0.09-1.65)$ & 0.205 \\
\hline
\end{tabular}

$\mathrm{CI}$, confidence interval; Other treatment, on treatment for other condition; Co_HIV, HIV co-infection. ${ }^{*} \mathrm{P}=<0.05$. 
knowledge gap by evaluating the independent risk factors for the previously established low quality of life scores in all domains of health as summarized in Tables 2-5. Common predictors of low scores in all the domains of quality of health studied namely physical, psychological, social relationships and environmental health are low monthly incomes and advancing duration of the illness. Apart from these common predictors of lower scores, advancing age and male gender predicts poor outcomes of psychological domain of health quality of life while the presence of co-morbidities or concomitant treatment for other conditions and unemployment are also predictive of lower quality of life scores in the physical domain of health. Wang et al. had reported alongside other factors that duration of illness affects the quality of life of tuberculosis patients using the SF-36 instrument in a Chinese population. ${ }^{21}$ This is in agreement with our present report showing duration of disease as a predictor of poor scores in all the domains.

Chang et al. in a review of the English literature had established the fact that a patient diagnosed with tuberculosis is less likely to find work or less likely to be able to work. ${ }^{6}$ This perhaps may explain our observation that unemployment is a predictor of low scores of physical health related quality of life. Perhaps, it will be more logical to suggest that the impairment of activities of daily living, declining work capacity, sapped energy and fatigue experienced by tuberculosis patient makes it less likely for them to be able to work optimally. This is further complicated by the stigma attached to this disease state in our environment and the fear of a spread in work environment that makes it less likely for such individuals to be gainfully employed. ${ }^{22}$ Unalan et al. while attempting to evaluate the differences in the quality of life between patients with active and inactive disease in an Indian population came to the conclusions that male gender, being single, a higher educational status and absence of concomitant diseases are associated with higher scores of quality of life using the SF-36 instrument, and that age is negatively correlated while monthly income is positively correlated to the quality of life of patients with active disease. ${ }^{23}$ While their study differs in design and objective from our present report and different instruments were employed, there are areas of agreement and disparities with our findings. The influence of the factors of age, monthly income and concomitant illnesses are in agreement with our findings while our study recorded higher scores for females in the psychological and social relationship domains as noted earlier for reasons earlier outlined.

\section{Conclusions}

Tuberculosis remains an important disease in Nigeria and the overall effect of the disease is all encompassing. Apart from being a significant cause of mortality and morbidity, its negative effects on the health related quality of life is enormous and might have been under-estimated and unattended. This study had identified some of the factors influencing the quality of life and independent predictors of low scores of quality of life in Nigerian black patients with tuberculosis. It therefore underscores the importance of instituting appropriate measures to improve these aspects of health related quality of life in our efforts towards optimizing care and treatment outcomes.

\section{References}

1. Centers for Disease Control and Prevention. HRQOL concepts. Available from: http:/www.cdc.gov/hrqol/concept.htm Accessed: 31 Jan 2012.

2. Sathvik RB, Parthasarathi G, Nahari MG. An assessment of the quality of life in haemodialysis patient using WHOQOLBREF questionnaire. Indian $\mathrm{J}$ Nephrol 2008;18:141-9.

3. Nirmal B, Divya KR, Dorairaj VS, Venkateswaran K. Quality of life in HIV/AIDS patients: a cross-sectional study in South India. Indian J Sex Transm Dis 2008;29:15-7.

4. Makanjuola AB, Adeponle AB, Obembe AO. A comparision of quality-of-life in schizophrenia and affective disorder patients in a Nigerian tertiary hospital. Transcult Psychiatry 2007;44:65-8.

5. Baba AI, Abdullah D, Olusegun B. The association between psychiatric disorders and quality of life of patient with diabetes mellitus. Iranian J Psychiatry 2007;2:30-4.

6. Chang B, Wu AW, Diette GB. Quality of life in tuberculosis: a review of the English language literature. Qual Life Res 2004;13: 1633-42.

7. Guo N, Marra CA, Marra F, et al. Health state utilities in latent and active tuberculosis. Value Health 2008;11:1154-61.

8. Guo N, Marra F, Marra CA. Measuring health- related quality of life in tuberculosis: a systematic review. Health Qual Life Outcomes 2009;7:14-23.

9. World Health Organization. Global tuberculosis control report 2007. Available from: http://www.who.int/tb/publications/global_r eport/archive/en/ Accessed: 31 Jan 2012.
10. World Health Organization. WHO Quality of Life-BREF (WHOQOL-BREF); 1997. Available from: http://www.who.int/substance abuse/research_tools/whoqolbref/en/ Accessed: 31 Jan 2012.

11. World Health Organization. WHOQOLBREF. Instructions, administration, scoring and generic version of the assessment; 1996. Available from: http://www.who.int/ mental_health/media/en/76.pdf Accessed: 31 Jan 2012.

12. Nigatu T, Abraha M. Epidemiological analysis of tuberculosis in Ethiopia: a ten year trend analysis. East Afr J Public Health 2010;7:182-6.

13. Sharma P, Jain S, Bamazai RNK, et al. Increased prevalence of pulmonary tuberculosis in male adults of Sahariya tribe of India: a revised survey. Indian $\mathrm{J}$ Community Med 2010;35:267-71.

14. Sharma PP, Kumat A, Sighn P. Sex differentials in the presentation of tuberculosis based on NFHS-2 and NFHS-3 data. Indian J Community Med 2010;35:230-7.

15. Lawson L, Lawson J0, Olajide I, et al. Sex differences in the clinical presentation of urban Nigerian patients with pulmonary tuberculosis. West Afr J Med 2008;27:82-6.

16. Muniyandi M, Rajeswari R, Balasubra-manian R. Evaluation of post treatment health related quality of life (HRQoL) among tuberculosis patient. Int J Tuberc Lung Dis 2007;11:887-92.

17. Miller MA. Culture, spirituality, and women's health. J Obstet Gynecol Neonatal Nurs 1995;24:257-63.

18. Walter T. Why are most churchgoers women? A literature review. Vox Evangelica 1990;20:73-90.

19. Dhuria M, Sharma N, Singh NP, et al. A study on the impact of tuberculosis on the quality of life and the effect after treatment with dots. Asia Pac J Public Health 2009;21:312-20.

20. Chamla D. The assessments of patients' health related quality of life during tuberculosis treatment in Wuhan, China. Int $\mathrm{J}$ Tuberc Lung Dis 2004;8:1100-6.

21. Wang Y, Lii J, Lu F. Measuring and Assessing the quality of life of patients with pulmonary tuberculosis. Chin J Tuberc Resp Dis 1998;21;700-3.

22. Abioye IA, Omotayo MO, Alakija W. Sociodemographic determinants of stigma among patients with pulmonary tuberculosis in Lagos, Nigeria. Afr Health Sci 2011;11:S100-4.

23. Unalan D, Soyuer F, Ceyhan 0, et al. Is the quality of life different in patients with active and inactive tuberculosis? Indian $\mathrm{J}$ Tuberc 2008;55:127. 